\section{Artificial intelligence} for air quality

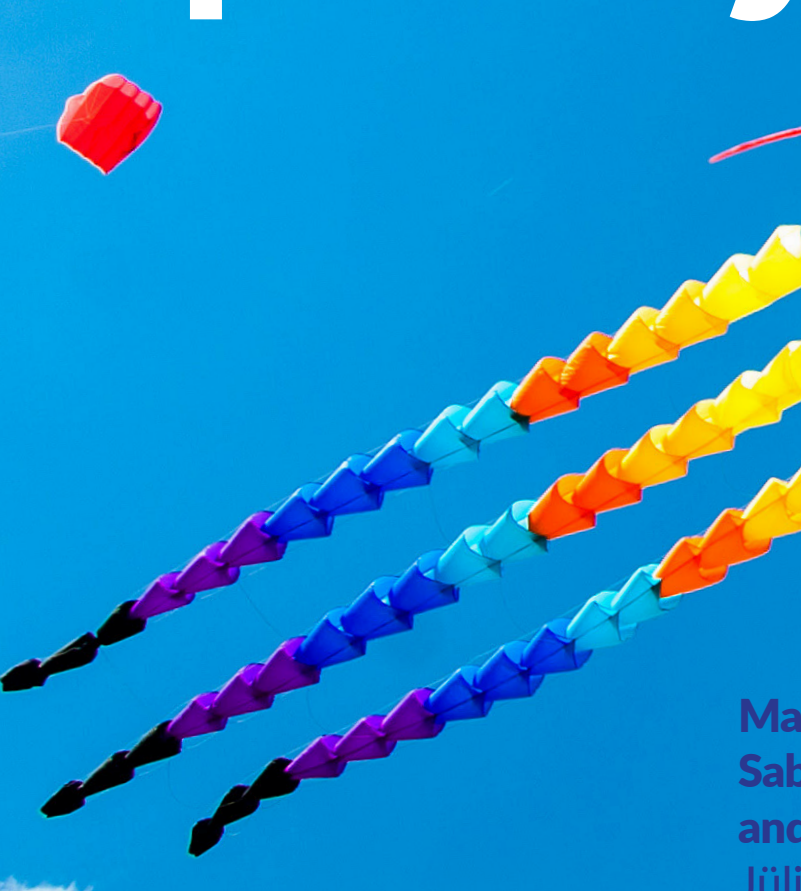

Jülich Supercomputing Centre, Research Centre Jülich,

Artificial intelligence (AI) is experiencing a wave of enthusiasm since ground-breaking results have been published on

cognitive problems such as image and speech recognition,

automated language translation, robotics, and strategic games.
This has become possible because of recent advances in massive dat processing capabihties (big data) and the development of deep learning (DI) neural network architectures, which 'learn' millons of parameters. Even though machine learning, in general, has been in use for many years, the uptake of DL in envionger slow, and IntelliAQ is one of the firt projects in the atmospheric sciences to fusy embrace the potential of modern bis aata processing and DL. ITtelliQ aims at shifting the analysis of global air pollutant observations to a new level and wi provide a basis for the future development of innovative air quality services with

Since the start of the IntelliAQ project in 2018, the development of machin learning solutions in all areas of environmental sciences has exploded Long-term doubts that deep neura networks might not be able to faithfuly represent the complex, multi-dimension and multi-scale patterns embedded in environmental data have been-replaced with a cautious optimism inspired by recent breakthroughs, especially in the area of weather forecasting (e.g. Scher Messori, 2019; Weyn, Durran \& Caruan 2020). Numerous studies are exploring state-of-the-art machine learning techniques for analysing weather and climate data (e.g. Callaghan et al., 2021) or developing new approaches to weather forecasting (e.g. Gong et al., 2021, submitted). In a recent position paper, my team and I tried to connect the state-of the-art in meteorological forecasting with the state-of-the-art machine learning and asked the question, "Can deep learnin beat numerical weather prediction? (Schultz et al., 2021)

Air pollution is in many ways closely related to weather. From a data science perspective, both applications deal with spatiotemporal patterns and nonGaussian data distributions, and the geometries and formats of meteorologic and air pollution datasets are quite similar. Air quality is determined by severa factors, including air pollutant emissions, chemical transformations, transport processes and weather. To analyse and understand air quality data and assess changes in air pollution levels, all of these factors must be taken into account. Air pollutant concentrations exhibit complex, The-dependent spatial patterns. Therefore, complex $\mathrm{DL}$ architectures
and comprehensive datasets are needed when we want to use Al for the analysis of when we want to use Al for the analysis of air quality and build air quality predictions based on modem machine learning. Furthermore, it is important to evaluate the machine learning results with proper develon a las. Meteorologists have metrics that differ frem the stater metrics that difer from the standard learning applications such as language or image processing.

The IntelliAQ project has positioned itself at the forefront of deep learning for the is of air quality information on the global scale. Specifically, IntelliAQ has three main objectives:

to develop novel spatial and temporal interpolation methods using deep neural networks in order to expand the coverage of historic and recent data while preserving fine-scale structures down to the street level

to develop an innovative air quality forecasting concept based on deep learning

to explore the use of deep neural networks to assess the quality of air pollution data and establish new, robust techniques for automated outlier detection and data screening.

Our research has primarily addressed tropospheric ozone, which is the second most important air polutant with adverse impacts on human health (WHO, 2021) vegetation (e.g. Unger et al., 2020) and climate (IPCC, 2021). A central asset of the project is the world's largest collection of ground-level ozone dat the database of the Tropospheric Ozone Assessment Report hosted at the Julich Supercomputing Centre, the home of IntelliAQ (Schultz et al., 2017). To account for the complexity of the multi-scale spatial and temporal interactions of tropospheric ozone, we have structured our research according to three main conceptual lines (Figure 1 . In the following, I will summarise what we achieved in each of these areas. To conclude, I will then provide a glimpse tho our plans to bring these three lines together and build one large, coherent thalysis and forecasting system for 21 months of the project.
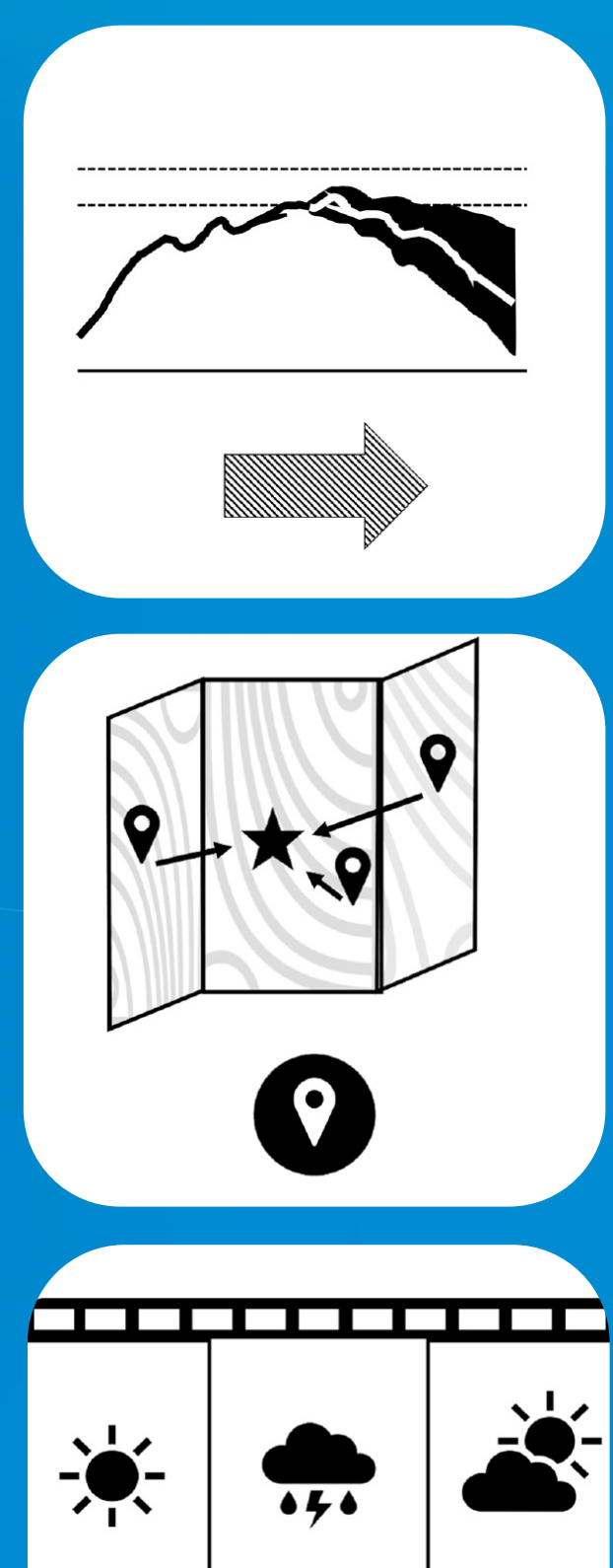
140

عصבصבصבص

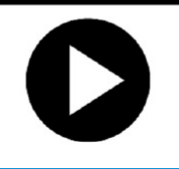
three methodological approaches pursued in spatial interpolation (mapning) and benttom: the application of advanced video prediction models to capture spatial and temporal patterns. 


\section{Time series analysis}

In light of the scattered geographic locations of air quality measurement sites and the available multi-variate long-term observation series from the TOAR database, the analysis of time series data, and specifically the attempt to forecast ozone concentrations with choice when the IntelliAQ project began. We explored different machine learnin concepts and have investigated severa strategies for the preparation of input data to test the limits of a purely datblock architecture and trained a single neural network with ten years of daily observations from around 300 German air quality monitoring sites to forecast daily maximum eight-hour average ozone concentrations over four days into the future. The model made use of information from nine variables (three chemical and six meteorologica generalisation and reasonable forecast
skill. It clearly outperformed a simple skill. It clearly outperformed a simple
ordinary least square regression model, driven approach for this task.

Kleinert et al. (2021) used an inception parameters) and showed very good

persistence forecast and a climatological values in the ozone prediction. This had forecast. At the time of publication, this mixed success because the better hit rate

was the largest neural network that of air quality threshold exceedances was has been trained with air quality data. Hower, some deficits of the neura strons two day and the of forecast quality after concentrations near average values. The latter implied that peak air pollution would rarely be predicted correctly.

To improve on the achievable forecast length, Leufen, Kleinert and Schultz (2021, submitted) explored the use of time filters and hourly observations as inputs, which yielded a substantial improvement (Figure 2). Kleinert et al. (manuscript in preparation) looked at ways to incorporate upstream information from sites that have seen the same air mass earlier. They developed a wind sector approach. This was first tested on gridded data from a chemistry transport model to avoid extra complication
when dealing with irregularly space when dealing with irregularly spaced
and missing data values. The sector and missing data values. The sector
approach also led to improved forecasts approach also led to improved forecasts over a longer period. Finally, a master approaches to better capture exper

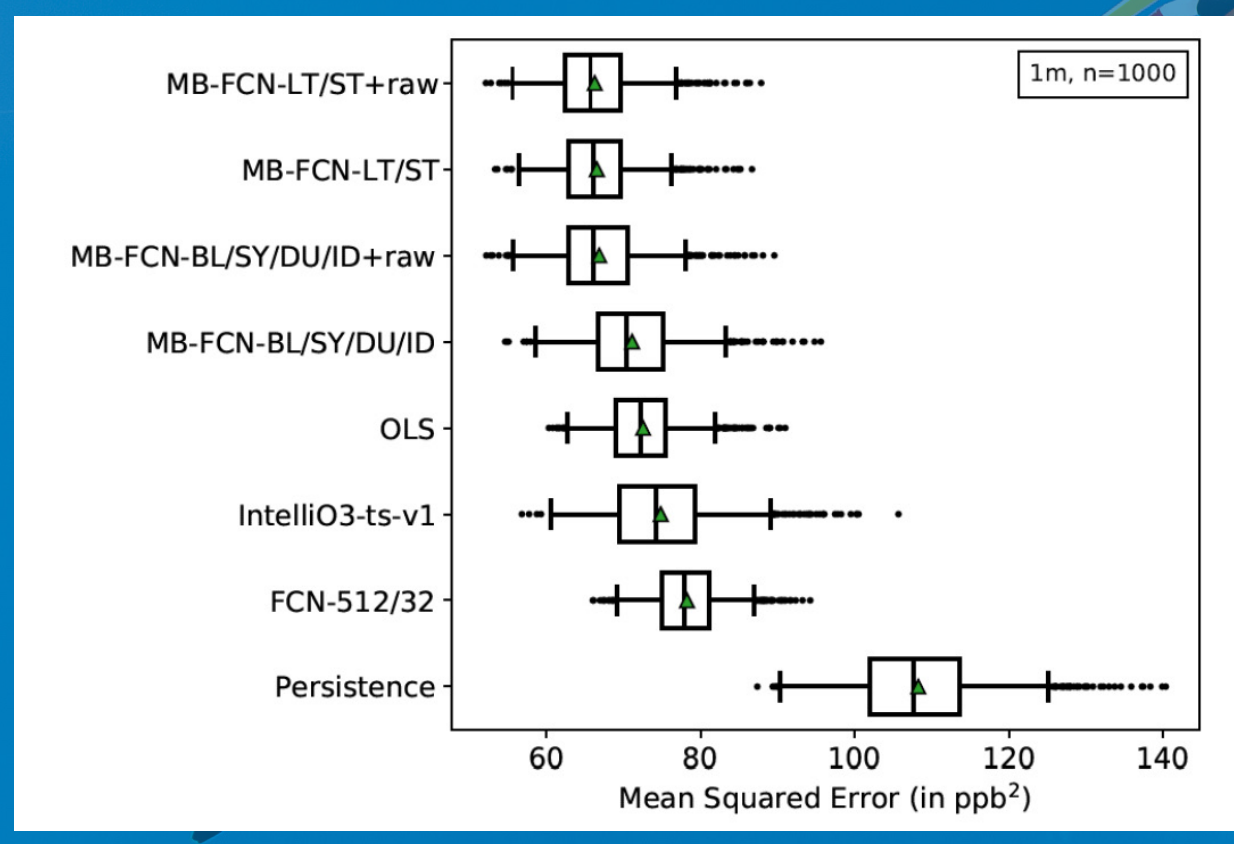

Figure 2: Error metrics (mean squared error) of different neural network and reference models predicting the daily maximum 8-hour ozone average concentrations at 32 air quality monitoring
sites in Northern Germany (training was performed on the data from 55 sites). Lower MSE values are better. The top four boxes and whiskers are results from a model with time filtering. OLS is a
linear least square model and IntelliO3-ts-v1 is the original model described in Kleinert, Leufen and linear least square model and IntelliO3-ts-t1 is the original model described in Kleinert, Leufen and
Schultz (2021). FCN-512/32 is a feed forward network without time filtering and Persistence is a Schultz (2021). FCN-512/32 is a feed forward network without time filtering and Persistence is a
persistence forecast, simply repeating the last values. Figure from Leufen, Kleinert \& Schultz, 2021 ,
submitted.
A user-friendly, adaptable software tool for time series forecasting through deep learning was developed and published together with the source code (Leufen, Kleinert \& Schultz, 2021). Future work on time series forecasting will look into making use of meteorological predictions (hstead of prescribing only data unti (now) and of a rich variety of geospatial data that has bech exploited in the spatia

\section{Mapping}

Here, we tested the use of a wide variety of freely available geospatial datasets (e.g. population density, digital elevation models, nighttime light intensity, landcover classes) to infer annual ozone average concentrations at locations without monitoring sites. random forests has been developed, where the high-resolution geospatial datasets serve as predictors for the air quality metrics calculated from the TOAR database (Betancourt et al., 2021). The study showed that about 60 per cent of the ozone variability can be explained by the geospatial predictors. In a follow-up study (Betancourt et al., in preparation), the mapping approach was extended across the whole globe (Figure 3). Recent techniques which make machine learning explainable (e.g. Lundberg \& Lee, 2017; Meyer \& Pebesma, 2020) were employed and further improved to assess the robustness and credibility of the generated global ozone concentration maps. The combination of these techniques is unique and can serve as a blueprint for future mapping studies with machine learning, which are not limited to air quality-related topics (Stadtler et al., 2021, submitted).

\section{Video prediction}

Initial attempts to apply relatively simple video prediction methods based on convolutional neural networks to forecasting spatiotemporal weather A novel mapping method based on

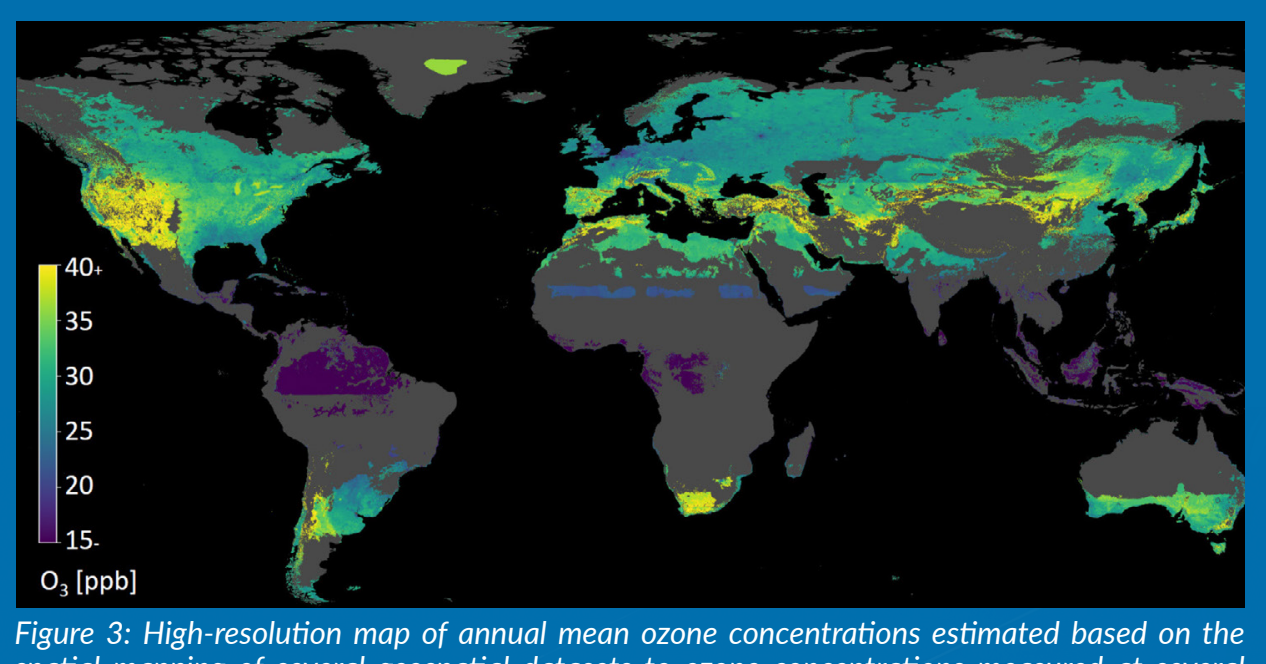

Figure 3: High-resolution map of annual mean ozone concentrations estimated based on the
spatial mapping of several geospatial datasets to ozone concentrations measured at several spatial mapping of several geospatial datasets to ozone concentrations measured at several
thousands of stations. Grey areas are regions where explainable Al techniques indicated too little
confidence in the extrapolation results. Figure from Betancourt et al., manuscript in preparation.

fields yielded unsatisfactory results. The resulting fields looked rather blurry, and important features (i.e. gradients in the data) were lost. This has lec to the adoption of generator models (Goodfellow et al., 2014), which draw samples from a learned distribution an can therefore produce sharp and realistic looking images.

In Gong et al. (2021, submitted), we In Gong et al. (2021, submitted), we variational autoencoder model (SAVP, Lee et al., 2018) for use as a meteorological et fal, 2018) for use as a meteorologica trained on 11 years of reanalysis data and generated skilful predictions of the 2-metre temperature over Europe over 12 hours. Even though the model was only hours. Even though the model was only
trained with temperature at two metres trained with temperature at two metres and at $850 \mathrm{hPa}$, it clearly outperformed a simpler convolutional LSTM model and a persistence forecast. However, the SAVP model exhibited some difficulties in correctly predicting the temperatures over mountains, and the model error is
still substantially larger than that of statestill substantially larger than that of stateof-the-art weather prediction models. W expect that additional input variables and an embedding of the surface orography would lead to improved forecasts, but to beat numerical weather prediction, one would also have to find ways to
incorporate some physical knowledge into the machine learning tools.

\section{Synthesis}

The different deep learning approaches described above have led to valuable insights with respect to the preparation of input data, the strengths and weaknesses and the technical hurdles to implement and train complex deep learning models on high-end supercomputer systems. is to design a deep learning model which can either generate trustworthy maps of global air pollution based on that are available or produce skilful over several days. It appears that socalled transformer models (e.g. Vaswani et al., 2017; Dosovitskiy et al., 2020) could be trained to learn spatiotemporal representations of atmospheric data and then be employed in various ways to accomplish the interpolation and transformer models belong to the class of terning systems, and they require substantial skill and computing power to achieve competitive results. We accept the challenge and hope that we can bring deep learning for state-of-the-art chemistry transport models in a variety of applications.

R References of different deep learning architectures the heterogeneous and scattered data forecasts of air polutant concentrations forecasting tasks that were defined in the IntelliAQ proposal. Such
IntelliAQ

PROJECT NAME

IntelliAQ

PROJECT SUMMARY

IntelliAQ is an ERC Advanced Grant project

to explore the application of cutting-edge machine learning techniques to global
air quality data in combination with high resolution geospatial and weather data. It combines novel data management and data science approaches to build the foundation
for innovative air quality information services.

PROJECT PARTNERS

The IntelliAQ project is hosted by
the Jülich Supercomputing Centre at Forschungszentrum Jülich and benefits from
Fon the world-class high-performance computing infrastructure at this institution. IntelliAQ has strong ties to the international Tropospheric Ozone Assessment Report activity and thus involves broad scientific collaboration.

PROJECT LEAD PROFILE

Dr Schultz works at the interface between atmospheric and computer science. He obtained his PhD at Forschungszentrum
Jülich in 1995 and worked at Harvard
University and the Max Planck Institute for Meteorology before he returned to Jülich in 2006. Since 2017 he established the research group Earth System Data Exploration, which develops new machine learning methods for Earth system science at the Jülich Supercomputing Centre.

CONTACTS

Dr Martin G. Schultz

Julch Supercomputing Centre,

entrum Jülich, 52425 Jülich.

II +4924616196870

凶.schultz@fz-juelich.de

(1) https://go.fzj.de/martinschultz

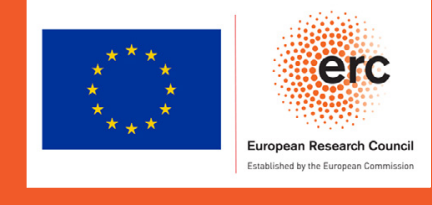

FUNDING

This project has received funding from the
European Research Council (ERC) under the European Research Council (ERC) under the
European Union's Horizon 2020 research and
innovat innovation programme under grant agreement
No.787576. 